\title{
Uniportal video-assisted thoracoscopic surgery: Sichuan Cancer Hospital experience
}

\author{
Huachuan Zhang, Run Xiang, Jintao He, Jiang Zhu \\ Department of Thoracic Surgery, Sichuan Cancer Hospital, Affiliate to the School of Medicine, The University of Electronic Science and \\ Technology of China, Chengdu 610041, China \\ Correspondence to: Jiang Zhu. Department of Thoracic Surgery, Sichuan Cancer Hospital, Affiliate to the School of Medicine, The University of \\ Electronic Science and Technology of China, Chengdu 610041, China. Email: 13008148289@163.com.
}

Submitted Sep 01, 2018. Accepted for publication Dec 13, 2018.

doi: $10.21037 /$ jtd.2018.12.76

View this article at: http://dx.doi.org/10.21037/jtd.2018.12.76

\section{Introduction}

The technique of uniportal video-assisted thoracic surgery (VATS) has developed and spread rapidly. More and more centers have been applying this technique to pulmonary wedge resection and lobectomy, following systematic lymphadenectomy and other complex thoracic surgery. Here, we present a surgery of left inferior lobectomy and systematic lymphadenectomy using uniportal videoassisted thoracic surgery. Through these examples, we hope we can contribute our experience towards an improved understanding of lobectomy and lymphadenectomy of advanced stage lung cancer.

\section{Clinical vignette}

A 57-year-old male patient who had a smoking history of 20 years and had smoked an average of 20 cigarettes a day presented with a history of persistent cough for one month. After he was admitted to our hospital, preoperative examination was performed. A computed tomography scan of the chest, abdomen and head demonstrated that a peripheral pulmonary nodule measuring $2.5 \mathrm{~cm} \times 2.0 \mathrm{~cm}$ was located in the lower lobe of left lung with enlarged interlobar and mediastinal lymph nodes on the ipsilateral side. Other preoperative examinations had not demonstrated any surgical contraindication. After thorough evaluation, we conducted a uniportal VATS lobectomy and systematic lymphadenectomy (Figure 1).

\section{Surgical techniques}

The patient received general anesthesia and double-lumen endobronchial intubation with single lung ventilation. The patient was then put in the right lateral decubitus position. The VATS procedure was performed by making a 4-cm single incision at the $5^{\text {th }}$ intercostal space at the left midaxillary line. A 30-degree 10-mm thoracoscopy was placed in the posterior part of the incision by a selfmaking latex glove ring. Other operating instruments were introduced into the incision simultaneously. The surgeon stood in front of the patient and the assistant was on the other side. We explored the chest cavity and found that the lesion was in the dorsal segment of the inferior lobe of the left lung which had evident pleural indentation. Combined with preoperative examination and operative exploration, we decided to perform left inferior lobectomy.

Firstly, we dissected the fissure and exposed the basilar segmental arteries and inferior lingular artery. Inferior to the bifurcation of the two arteries, an enlarged interlobular lymph node was detected. It was too difficult to dissect the lymph node from the arteries, so we had to transect the inferior lingular artery with vascular clips. The left inferior pulmonary artery was mobilized and then divided with an endoscopic stapler. Secondly, we dissected the inferior pulmonary ligament and mobilized the inferior pulmonary vein. We then divided it with an endoscopic stapler. Posterior to the left pulmonary artery, the left inferior bronchus was exposed and divided again with a stapler. All 


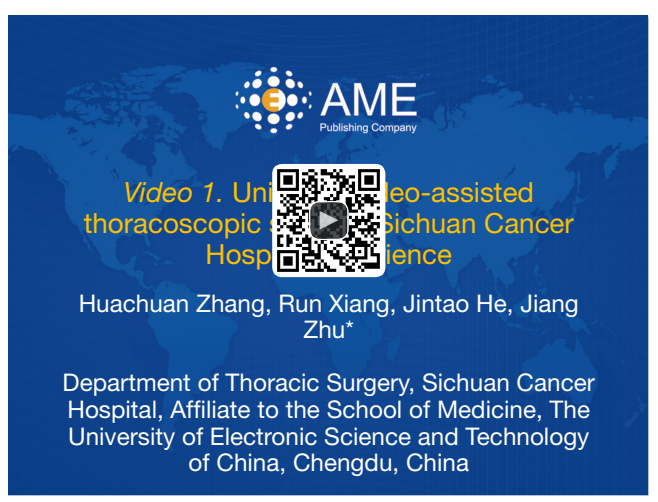

Figure 1 Uniportal video-assisted thoracoscopic surgery: Sichuan Cancer Hospital experience (1).

Available online: http://www.asvide.com/article/view/29636

hilar structures were transected using 60-mm endoscopic staplers.

The resected lobe was removed with a protective bag. At last, we performed systematic lymphadenectomy including the left lower paratracheal, subaortic, para-aortic, subcarinal, pulmonary ligament, and hilar lymph nodes. A 26-Fr chest tube was placed in the anterior part of the utility incision. Total duration of the procedure was 97 minutes, and the estimated blood loss was $100 \mathrm{~mL}$.

Postoperative recovery of the patient was successful and satisfactory. The chest tube was removed on postoperative day (POD) 3. The patient was discharged on the POD 5 with no complications. Subsequent pathological examination revealed moderately differentiated adenocarcinoma with subaortic, subcarinal, and interlobar lymph node involvement.

\section{Comments}

With the development of the minimally invasive concept, the technique of video-assisted thoracic surgery has changed dramatically in recent years. Nowadays, with its increasing proliferation and establishment in centers around the world, it can be said without exaggeration that uniportal video-assisted thoracic surgery represents the new frontier of minimally invasive thoracic surgery. It has enjoyed popular support both from the newest generation of thoracic surgeons who are more receptive to innovative solutions, and from the chest physicians who are keen to consider surgical treatment for their patients (2). UniVATS has evident advantages such as a single, minimal and more cosmetic skin incision, less postoperative pain and discomfort, shorter hospital stay, and greater patient acceptance and satisfaction of the thoracic operation. It has become the biggest technological innovation in the field since VATS itself emerged $(3,4)$. Uni-VATS procedures, such as lung wedge resection and lobectomy, have been a conventional procedure for patients of early stage nonsmall-cell lung cancer and elderly patients of lung cancer. Other complex uniportal VATS lung resections including pneumonectomy (5), segmentectomy (6), bronchial sleeve lobectomy (7), lobectomy with pulmonary artery reconstruction $(8)$, and double sleeve lobectomy $(9,10)$ have been performed and published in succession.

Our center has been performing uniportal VATS since 2015 . We retrospectively analyzed the clinical and pathological data of the 133 cases on whom we performed uniportal VATS from March 2015 to October 2016. There were 79 cases of male patients and 54 cases of female patients, while the mean age was 52.21. There were 6 conversions to thoracotomy. In the 127 cases of completed uniportal VATS, there were 78 lobectomies, 6 anatomical segmentectomies, 30 wedge resections, and 6 pleurectomy biopsies. All patients were successfully discharged without serious postoperative complication or death. Clinical outcome of uniportal VATS compared with multiportal VATS was investigated. Patients in the multiportal group had smaller surgical incisions than those in the uniportal group. Pain scores of the uniportal group and multiportal group were $4.18 \pm 1.67$ and $6.54 \pm 1.83$ within 24 hours after operation, and $3.05 \pm 1.47$ and $4.68 \pm 1.64$ within 48 hours after operation, respectively. Operation data, postoperative complications and hospital stay were similar in both groups. As can be seen, the thoracic surgeon who has mastered the technique of multiportal VATS proficiently could easily and skillfully grasp the technique of uni-VATS lobectomy with the learning curve of uni-VATS.

At the same time, the speedy development of the uniVATS technique has enlarged the indications and reduced the contraindications (2). Controversies have emerged about the application of uni-VATS in both the advanced stages of lung cancer and mediastinal lymphadenectomy that is essential in lung cancer surgery $(11,12)$. In the above case, not only interlobular lymph nodes but also some mediastinal lymph nodes of the patient were enlarged and proved positive by the final postoperative pathological report. So, with a surgeon's accumulated experience in uni-VATS, this surgery can be safe and is warranted in being generalized for advanced stages of lung cancer and systematic lymphadenectomy. Now, with the development 
of the endoscopic device and surgery instrument, uni-VATS will be applied in more and more surgeries that were once considered to be contraindications.

\section{Acknowledgements}

None.

\section{Footnote}

Conflicts of Interest: The authors have no conflicts of interest to declare.

Informed Consent: Written informed consent was obtained from the patient for publication of this manuscript and any accompanying images.

\section{References}

1. Zhang H, Xiang R, He J, et al. Uniportal videoassisted thoracoscopic surgery: Sichuan Cancer Hospital experience. Asvide 2019;6:021. Available online: http:// www.asvide.com/article/view/29636

2. Mineo TC, Ambrogi V. A glance at the history of uniportal video-assisted thoracic surgery. J Vis Surg 2017;3:157.

3. Rocco G, Martin-Ucar A, Passera E. Uniportal VATS wedge pulmonary resections. Ann Thorac Surg 2004;77:726-8.

4. Jutley RS, Khalil MW, Rocco G. Uniportal vs standard three-port VATS technique for spontaneous pneumothorax: comparison of post-operative pain and residual paraesthesia. Eur J Cardiothorac Surg 2005;28:43-6.

Cite this article as: Zhang $\mathrm{H}$, Xiang R, He J, Zhu J. Uniportal video-assisted thoracoscopic surgery: Sichuan Cancer Hospital experience. J Thorac Dis 2019;11(1):273-275. doi: 10.21037/ jtd.2018.12.76
5. Gonzalez-Rivas D, de la Torre M, Fernandez R, et al. Singleport video-assisted thoracoscopic left upper lobectomy. Interact Cardiovasc Thorac Surg 2011;13:539-41.

6. Gonzalez-Rivas D, Mendez L, Delgado M, et al. Uniportal video-assisted thoracoscopic anatomic segmentectomy. J Thorac Dis 2013;5 Suppl 3:S226-33.

7. Gonzalez-Rivas D, Fernandez R, Fieira E, et al. Uniportal video-assisted thoracoscopic bronchial sleeve lobectomy: first report. J Thorac Cardiovasc Surg 2013;145:1676-7.

8. Gonzalez-Rivas D, Delgado M, Fieira E, et al. Single-port video-assisted thoracoscopic lobectomy with pulmonary artery reconstruction. Interact Cardiovasc Thorac Surg 2013;17:889-91.

9. Gonzalez-Rivas D, Delgado M, Fieira E, et al. Double sleeve uniportal video-assisted thoracoscopic lobectomy for non-small cell lung cancer. Ann Cardiothorac Surg 2014;3:E2.

10. Gonzalez-Rivas D, Fieira E, Delgado M, et al. Uniportal video-assisted thoracoscopic sleeve lobectomy and other complex resections. J Thorac Dis 2014;6:S674-81.

11. Delgado Roel M, Fieira Costa EM, González-Rivas D, et al. Uniportal video-assisted thoracoscopic lymph node dissection. J Thorac Dis 2014;6:S665-8.

12. Liu CC, Shih CS, Pennarun N, et al. Transition from a multiport technique to a single-port technique for lung cancer surgery: is lymph node dissection inferior using the single-port technique?†. Eur J Cardiothorac Surg 2016;49 Suppl 1:i64-72.

(English Language Editor: John Ayric Gray, AME Publishing Company) 